\title{
The Self-Similarly Expanding Eshelby Ellipsoidal Inclusion: I. Field Solution
}

\author{
Luqun $\mathrm{Ni}^{\mathrm{a}}$, Xanthippi Markenscoff ${ }^{\mathrm{a}, *}$ \\ ${ }^{a}$ Department of Mechanical and Aerospace Engineering \\ University of California, San Diego \\ La Jolla, California, 92093-0411
}

\begin{abstract}
The solution of a self-similarly (subsonically) dynamically expanding ellipsoidal inclusion with general spatially uniform transformation strain temporally constant is obtained by the use of the Radon transform and the satisfaction of the initial conditions and the radiation condition at infinity. It constitutes the self-similar evolution of the inclusion singularity (jump discontinuity at the inclusion boundary) starting from zero dimension. The field solutions for the displacement gradient and particle velocity are presented. Due to the fact that for a self-similarly expanding subsonic motion the hyperbolic system of the partial differential equations of motion becomes elliptic (as proved in Ni and Markenscoff, 2015), it is shown here explicitly that the solution for the displacement gradient in the interior domain of the expanding ellipsoid is constant, thus extending the Eshelby property to the self-similarly expanding ellipsoids as pointed out by Burridge and Willis (1969). Also, the particle velocity is shown to be zero in the interior domain (lacuna) as the waves emitted by the self-similarly expanding inclusion cancel each other due to the symmetries of the motion and geometry.
\end{abstract}

Key words: A. Elastodynamics, B. transformation strain, C. self similarity, D. ellipsoidal inclusion 


\section{Introduction}

One of the seminal solutions of the twentieth century solid mechanics is the Eshelby (1957) ellipsoidal inclusion with uniform transformation strain that has the property of constant constrained strain in the interior domain. The Eshelby tensor relates the constrained strain to the transformation strain (or eigenstrain, a term introduced by Dundurs, 1967), the constancy of which in the interior domain allows for the analytical solutions of ellipsoidal inhomogeneities under uniform loading at infinity or transformation strain in the inhomogeneity (Eshelby, 1957, 1959, 1961), and which lead to the widespread application of the Eshelby tensor. More recently, Markenscoff and Ni (2010) have obtained the solution for a dynamically expanding spherical inclusion with dilatational transformation strain in general subsonic motion, and, by a limiting procedure as the radius of the expanding sphere tends to infinity, they obtained the radiated fields of a moving plane phase boundary (with inertia). The moving (expanding or shrinking) strip inclusion under general uniform eigenstrain, and the driving-force on each boundary have been obtained by Markenscoff and Ni (2011, 2012). Inclusion fixed in space with time dependent eigenstrain have been treated in the literature (e.g., Willis, 1965; Mikata and Nemat-Nasser, 1990; Michelitsch, et al, 2003). But the true dynamic generalization of the Eshelby tensor is this problem, where the constant stress Eshelby property is valid in dynamics too.

In this paper we analyze the self-similarly subsonically expanding ellipsoidal inclusion with general uniform transformation strain, temporally constant. Due to the fact that for a self-similarly expanding subsonic motion the hyperbolic system of the partial differential equations of motion becomes elliptic (as proved in $\mathrm{Ni}$ and Markenscoff, 2015), it is shown here by explicit solution for the displacement gradient that in the interior domain of the ex-

\footnotetext{
* Corresponding author.

Email addresses: Ini@ucsd.edu (Luqun Ni), xmarkens@ucsd.edu (Xanthippi Markenscoff).
} 
panding ellipsoid it is constant, thus extending the Eshelby property to the self-similarly expanding ellipsoid. The analysis of Burridge and Willis (1969) for a two-dimensional expanding self-similar elliptical crack when applied to phase change in an anisotropic solid would give the same Eshelby property, as pointed out in Burridge and Willis (1969).

In this paper we apply the Radon transform to the governing partial differential equations (Willis, 1973; Wang and Achenbach, 1994; Li and Wang, 2008) and invert it on the basis of the satisfaction of the initial conditions and the radiation condition at infinity to obtain the solution for the displacement of a self-similarly expanding ellipsoidal inclusion. The solution is in agreement with Burridge and Willis (1969) for self-similarly expanding ellipsoidal crack. The displacement gradient and particle velocity fields are obtained as well.

Due to the symmetries of the ellipsoidal geometry and the self-similarity of the motion, the inwardly emitted waves from the inclusion boundary cancel each other and this results in zero particle velocity in the interior domain (lacuna), which is obtained here by explicit calculation of the particle velocity. The zero particle velocity in the interior domain was shown recently by Markenscoff (2015) based on dimensional analysis and analytic arguments alone. It may be noted that for general non-self-similar motion, the waves emitted inwardly by the expanding inclusion are focussed at the center (with dilatational eigenstrain) (Markenscoff and Ni, 2010; Ni and Markenscoff, 2015) as they are for temporally varying eigenstrain within a spherical inclusion (Willis, 1965).

The applications of the dynamically expanding inclusion with transformation strain may include phenomena such as inducement of martensitic transformations under dynamically applied loading (Yang, et al., 2009). Yang, et al. (2009) used the static Eshelby inclusion solution in their modeling, but the above mentioned fields with inertia may be more appropriate. Expanding regions of transformation strain have application to geophysics and seismology (Rice, 1980, pp.557-56; Backus and Mulcahy, 1976), where the transformation 
strain concept is the fundamental basis for the concept of seismic moment which routinely quantifies the earthquake size in modern seismology, with the expanding inclusion and inhomogeneity being a model for the source of deep earthquakes (Burnley and Green, 1989, and Green and Burnley, 1989). Obviously, the energy for the expansion comes from the conversion of another source of energy, e.g., thermal and/or chemical. The constant stress Eshelby property for self-similarly expanding ellipsoidal inclusion and the determination of the constant interior Dynamic Eshelby Tensor (computed in Part II of this paper for the expanding sphere) allows for the solution of expanding inhomogeneities with transformation strain by the Eshelby equivalent inclusion method (Eshelby, 1957) extended now to dynamics. In Part II, the static Eshelby tensor for the sphere is obtained from the dynamic one by a limiting procedure in which the expansion velocities tend to zero and the time to infinity. The particle velocity jumps on the moving inclusion boundary are also obtained in Part II. Self-similar motion is important because it grasps the early response of the system before other interactions take place (Barenblatt, 1979, 1996).

\section{Governing equation for a self-similarly subsonically expanding ellipsoidal Eshelby inclusion}

We consider an ellipsoidal Eshelby inclusion (with transformation strain spatially and temporally uniform) centered at the origin of a Cartesian coordinate system $\left(x_{1}, x_{2}, x_{3}\right)$, with zero-dimension at time $t=0$ and expanding in a self-similar manner in an infinite linear elastic medium. At time $t$, the inclusion occupies the ellipsoid

$\left\{\boldsymbol{x}=\left(x_{1}, x_{2}, x_{3}\right) \mid s_{1}^{2} x_{1}^{2}+s_{2}^{2} x_{2}^{2}+s_{3}^{2} x_{3}^{2} \leq t^{2}\right\}$,

with semi-axes of magnitude $t / s_{1}, t / s_{2}$, and $t / s_{3}$, i.e., with axes expansion velocities $1 / s_{1}, 1 / s_{2}, 1 / s_{3}$, assumed subsonic. The uniform eigenstrain (the ter- 
minology introduced by Dundurs, 1967), nonzero in the interior of the expanding ellipsoid, is expressed as

$\epsilon_{l m}^{*}(\boldsymbol{x}, t)=\epsilon_{l m}^{*} H\left(t-\left(s_{r}^{2} x_{r}^{2}\right)^{1 / 2}\right)$,

where $H$ denotes the Heaviside step function, and, unless stated otherwise, the Latin indices here and throughout the paper are in the range 1,2,3. The summation convention is used throughout. The governing equations for this problem are (Willis, 1965; Markenscoff and Ni, 2010)

$\rho \frac{\partial^{2} u_{j}}{\partial t^{2}}-C_{j k l m} \frac{\partial^{2} u_{l}}{\partial x_{k} \partial x_{m}}=-C_{j k l m} \epsilon_{l m}^{*} \frac{\partial}{\partial x_{k}} H\left(t-\left(s_{r}^{2} x_{r}^{2}\right)^{1 / 2}\right)$,

with the initial conditions

$\boldsymbol{u}(\boldsymbol{x}, 0)=0$,

$\frac{\partial \boldsymbol{u}}{\partial t}(\boldsymbol{x}, 0)=0$,

and the radiation condition of all fields vanishing at infinity.

In the form of operators, the governing equations Eq. (2.3) are rewritten as $L_{j l}\left(\frac{\partial}{\partial t}, \nabla\right) u_{l}=-K_{j}(\nabla) H\left(t-\left(s_{r}^{2} x_{r}^{2}\right)^{1 / 2}\right)$,

where we defined the operators

$$
\begin{aligned}
& L_{j l}\left(\frac{\partial}{\partial t}, \nabla\right)=\rho \frac{\partial^{2}}{\partial t^{2}} \delta_{j l}-C_{j k l m} \frac{\partial^{2}}{\partial x_{k} \partial x_{m}}, \\
& K_{j}(\nabla)=C_{j k l m} \epsilon_{l m}^{*} \frac{\partial}{\partial x_{k}} .
\end{aligned}
$$

The governing equations (2.3) are invariant under the scalings of the variables $\boldsymbol{x}, t$, and the displacement $\boldsymbol{u}$. In fact, if $\boldsymbol{u}(\boldsymbol{x}, t)$ solves (2.3), and we define a scaling of uniform stretch of the variables, for $\alpha>0$,

$x_{i}^{*}=\alpha x_{i}, \quad t^{*}=\alpha t, \quad u_{i}^{*}=\alpha u_{i}(\boldsymbol{x}, t)$, 
then $\boldsymbol{u}^{*}$ satisfies the equations

$\rho \frac{\partial^{2} u_{j}^{*}}{\partial t^{* 2}}-C_{j k l m} \frac{\partial^{2} u_{l}^{*}}{\partial x_{k}^{*} \partial x_{m}^{*}}=-C_{j k l m} \epsilon_{l m}^{*} \frac{\partial}{\partial x_{k}^{*}} H\left(t^{*}-\left(s_{r}^{2} x_{r}^{* 2}\right)^{1 / 2}\right)$,

and the initial conditions

$\boldsymbol{u}^{*}\left(\boldsymbol{x}^{*}, 0\right)=\frac{\partial \boldsymbol{u}^{*}}{\partial t^{*}}\left(\boldsymbol{x}^{*}, 0\right)=0$

and the radiation condition at infinity.

The governing equations (2.3) and the initial and boundary conditions (2.4), (2.5), and (??) are thus invariant under the scaling of variables (2.9).

According to the general theory of dimensional analysis (Barenblatt, 1979, 1996; Bluman and Kumei, 1989), since the system of equations of motion (2.3) is scaling invariant, the number of the independent variables can be reduced by one, and it allows for a self-similar solution. To prove this explicitly, see e.g., Bluman and Kumei (1989), and Ni and Markenscoff (2015), since $\boldsymbol{u}(\boldsymbol{x}, t)$ satisfies Eqs. (2.3) and the initial conditions (2.4) and (2.5), and the radiation condition at infinity, it is clear that $\boldsymbol{u}\left(\boldsymbol{x}^{*}, t^{*}\right)$ solves Eqs. (2.10) (??). According to the uniqueness for an unbounded domain (Wheeler and Sternberg, 1968), it follows that $\boldsymbol{u}^{*}$ is identical to $\boldsymbol{u}\left(\boldsymbol{x}^{*}, t^{*}\right)$. Hence,

$\boldsymbol{u}\left(\boldsymbol{x}^{*}, t^{*}\right)=\boldsymbol{u}(\alpha \boldsymbol{x}, \alpha t)=\boldsymbol{u}^{*}=\alpha \boldsymbol{u}(\boldsymbol{x}, t)$.

We define that, for $t>0$,

$\boldsymbol{z}=\frac{\boldsymbol{x}}{t} \quad$ and $\quad \boldsymbol{\phi}(\boldsymbol{z}, t)=\frac{\boldsymbol{u}(\boldsymbol{x}, t)}{t}$,

then, we have

$\boldsymbol{u}(\alpha \boldsymbol{x}, \alpha t)=\alpha t \phi\left(\left(\frac{\alpha \boldsymbol{x}}{\alpha t}\right), \alpha t\right)=\alpha t \boldsymbol{\phi}(\boldsymbol{z}, \alpha t)$. 
From (2.12), (2.13) and (2.14), follows that

$\phi(\boldsymbol{z}, \alpha t)=\boldsymbol{\phi}(\boldsymbol{z}, t)$

Since $\alpha>0$ is arbitrary, it follows that $\phi$ is actually independent of $t$, and

$\boldsymbol{u}(\boldsymbol{x}, t)=t \boldsymbol{\phi}(\boldsymbol{z})$

Hence, $u_{l} / t, \partial u_{l} / \partial x_{i}=\partial \phi_{l}(\boldsymbol{x} / t) / \partial\left(x_{i} / t\right)$, and the components of the stress are homogeneous functions of $(\boldsymbol{x}, t)$ of order zero, and are hence self-similar.

Since the system of equations (2.3) is scaling invariant under scaling (2.9) of stretching coordinates, the self-similar solution of the displacement field is written in the new dependent variable $\phi$ and the new independent variable $\boldsymbol{z}=\boldsymbol{x} / t$ as

$u_{l}(\boldsymbol{x}, t)=t \phi_{l}\left(\frac{\boldsymbol{x}}{t}\right)=t \phi_{l}(\boldsymbol{z})$.

Thus, we have

$\frac{\partial \boldsymbol{u}}{\partial x_{m}}=\frac{\partial}{\partial x_{m}}(t \boldsymbol{\phi}(\boldsymbol{z}))=\frac{\partial \boldsymbol{\phi}}{\partial z_{m}}, \quad \frac{\partial^{2} \boldsymbol{u}}{\partial x_{k} \partial x_{m}}=\frac{1}{t} \frac{\partial^{2} \boldsymbol{\phi}}{\partial z_{k} \partial z_{m}}$.

Also, $\partial z_{m} / \partial t=-z_{m} / t$, and

$\frac{\partial \boldsymbol{u}}{\partial t}=\frac{\partial}{\partial t}(t \boldsymbol{\phi}(\boldsymbol{z}))=\boldsymbol{\phi}(\boldsymbol{z})-z_{m} \frac{\partial \phi}{\partial z_{m}}$,

$\frac{\partial^{2} \boldsymbol{u}}{\partial t^{2}}=\frac{z_{m} z_{k}}{t} \frac{\partial^{2} \boldsymbol{\phi}}{\partial z_{k} \partial z_{m}}$.

By using (2.17) - (2.20), the governing equations of elastodynamics (2.3) for a self-similarly subsonically expanding ellipsoidal inclusion, in terms of the new variables $\boldsymbol{z}$ and $\boldsymbol{\phi}$, reduce to a system of partial differential equations with three independent variables $z_{i}$,

$\left[\rho \delta_{j l}\left(z_{k} \frac{\partial}{\partial z_{k}}\right)\left(z_{m} \frac{\partial}{\partial z_{m}}\right)-C_{j k l m} \frac{\partial^{2}}{\partial z_{k} \partial z_{m}}\right] \phi_{l}=-C_{j k l m} \epsilon_{l m}^{*} \frac{\partial}{\partial z_{k}}\left[H\left(1-s_{k}^{2} z_{k}^{2}\right)\right]$. 
As proved in Ni and Markenscoff (2015), this system of partial differential equations is elliptic when $|\boldsymbol{z}|<\min \{a, b\}$, and hyperbolic otherwise, where

$a=\sqrt{(\lambda+2 \mu) / \rho}$ and $b=\sqrt{\mu / \rho}$ are the elastic compression and shear wave speeds, respectively; also for most elastic materials $a>b$, that is $a^{2}-b^{2}=$ $(\lambda+\mu) / \rho=(K+\mu / 3) / \rho>0$, here $K$ is the bulk modulus of the material. While there are materials having negative bulk modulus and such that $K+\mu / 3<0$, which leads to $a<b$, see e.g. Wang and Lakes (2005).

By using the operators defined in (2.7) and (2.8), we have

$$
\begin{aligned}
& L_{j l}\left(z_{m} \frac{\partial}{\partial z_{m}}, \nabla_{z}\right)=\rho\left(z_{m} \frac{\partial}{\partial z_{m}}\right)^{2} \delta_{j l}-C_{j k l m} \frac{\partial^{2}}{\partial z_{k} \partial z_{m}}, \\
& K_{j}\left(\nabla_{z}\right)=C_{j k l m} \epsilon_{l m}^{*} \frac{\partial}{\partial z_{k}},
\end{aligned}
$$

we rewrite the governing system of equations (2.21) as

$L_{j l}\left(\left(z_{k} \frac{\partial}{\partial z_{k}}\right), \nabla_{z}\right) \phi_{l}(\boldsymbol{z})=-K_{j}\left(\nabla_{z}\right) H\left(1-s_{k}^{2} z_{k}^{2}\right)$,

where $\nabla_{z}=\left(\partial / \partial z_{1}, \partial / \partial z_{2}, \partial / \partial z_{3}\right)$. In the sequel, the governing system of equations (2.24) will be solved by the Radon transform.

\section{Field solutions of the self-similarly subsonically expanding ellip- soidal inclusion in an isotropic medium}

\subsection{Solution by the Radon transform}

In order to solve the governing system of equations (2.24), we will use the Radon transform to build up $\phi$ from plane wave functions (John, 1955) which take constant values on the hyper-planes perpendicular to the vector $\boldsymbol{z}$ (and depend on the inner product of $\boldsymbol{z}$ and the Radon transform variable $\boldsymbol{\xi}$ ), so 
that the system of differential equations (2.24) will be reduced to a system of algebraic equations.

By using the Radon transform, Willis (1973) has solved various self-similar problems in elastodynamics, also see Wang and Achenbach (1994) and Li and Wang (2008). The Radon transform of $\boldsymbol{\phi}$ is defined by

$\hat{\boldsymbol{\phi}}(s, \boldsymbol{\xi})=\mathcal{R}(\boldsymbol{\phi}(\boldsymbol{z}))=\int_{R^{3}} \boldsymbol{\phi}(\boldsymbol{z}) \delta(s-\boldsymbol{z} \cdot \boldsymbol{\xi}) d \boldsymbol{z}$,

where $s \in R^{1}, \boldsymbol{\xi}$ is a unit vector in $R^{3}$, and $\boldsymbol{z} \cdot \boldsymbol{\xi}=z_{k} \xi_{k}$ is the inner product of $\boldsymbol{z}$ and $\boldsymbol{\xi}$ (see, e.g., Ludwig, 1966; Willis, 1973; Wang and Achenbach, 1994; $\mathrm{Li}$ and Wang, 2008). By the inverse Radon transform of $\hat{\phi}, \phi$ is given as

$\phi(\boldsymbol{z})=\mathcal{R}^{-1}(\hat{\boldsymbol{\phi}})=-\left.\frac{1}{8 \pi^{2}} \int_{|\boldsymbol{\xi}|=1} \partial_{s}^{2} \hat{\boldsymbol{\phi}}(s, \boldsymbol{\xi})\right|_{s=(\boldsymbol{z} \cdot \boldsymbol{\xi})} d S$,

where $S=\{\boldsymbol{\xi}|| \boldsymbol{\xi} \mid=1\}$ is the unit sphere in $R^{3}$.

Substituting Eq. (3.1) into the system of governing equations (2.24), we obtain

$\left.\int_{|\boldsymbol{\xi}|=1}\left[L_{j l}\left(s, \xi_{m}\right) \partial_{s}^{4} \hat{\phi}_{l}(s, \boldsymbol{\xi})\right]\right|_{s=(\boldsymbol{z} \cdot \boldsymbol{\xi})} d S=-\left.\int_{|\boldsymbol{\xi}|=1} K_{j}(\boldsymbol{\xi}) \partial_{s}^{3} \hat{H}(s, \boldsymbol{\xi})\right|_{s=(\boldsymbol{z} \cdot \boldsymbol{\xi})} d S$

where, from (2.23),

$K_{j}(\boldsymbol{\xi})=C_{j k l m} \epsilon_{l m}^{*} \xi_{k}$,

and $\hat{H}(s, \boldsymbol{\xi})$ is the Radon transform of the step function $H\left(1-s_{k}^{2} z_{k}^{2}\right)$ given by

$\hat{H}(s, \boldsymbol{\xi})=\mathcal{R}\left(H\left(1-s_{k}^{2} z_{k}^{2}\right)\right)=\int_{R^{3}} H\left(1-s_{k}^{2} z_{k}^{2}\right) \delta\left(s-z_{k} \xi_{k}\right) d z$.

Then, it suffices to solve $\hat{\phi}_{l}(s, \boldsymbol{\xi})$ from the system of equations

$L_{j l}(s, \boldsymbol{\xi}) \partial_{s}^{4} \hat{\phi}_{l}(s, \boldsymbol{\xi})=-K_{j}(\boldsymbol{\xi}) \partial_{s}^{3} \hat{H}(s, \boldsymbol{\xi})$, 
which is a system of algebraic equations for the unknown functions $\partial_{s}^{4} \hat{\phi}_{l}(s, \boldsymbol{\xi})$ for $|\boldsymbol{\xi}|=1$ and $s=(\boldsymbol{z} \cdot \boldsymbol{\xi}), \boldsymbol{z} \in R^{3}$.

In terms of the co-factor matrix and the determinant, inverting the matrix $L_{j l}(\gamma, \boldsymbol{\xi})$ for subsonic motion, we have

$L_{l j}^{-1}(\gamma, \boldsymbol{\xi})=\frac{N_{l j}(\gamma, \boldsymbol{\xi})}{D(\gamma, \boldsymbol{\xi})}$,

where in an isotropic medium,

$$
\begin{aligned}
& N_{l j}(\gamma, \boldsymbol{\xi})=\rho\left[\left(\gamma^{2}-a^{2}|\boldsymbol{\xi}|^{2}\right) \delta_{j l}+\left(a^{2}-b^{2}\right) \xi_{j} \xi_{l}\right], \\
& D(\gamma, \boldsymbol{\xi})=\rho^{2}\left(\gamma^{2}-b^{2}|\boldsymbol{\xi}|^{2}\right)\left(\gamma^{2}-a^{2}|\boldsymbol{\xi}|^{2}\right) .
\end{aligned}
$$

Applying $N_{l j}$ to both sides of (3.6), we have

$D(s, \boldsymbol{\xi}) \partial_{s}^{4} \hat{\phi}_{l}(s, \boldsymbol{\xi})=-N_{l j}(s, \boldsymbol{\xi}) K_{j}(\boldsymbol{\xi}) \partial_{s}^{3} \hat{H}(s, \boldsymbol{\xi})$.

We can write the solution of (3.10) as

$\partial_{s}^{4} \hat{\phi}_{l}=\Phi_{l}^{g}+\Phi_{l}^{p}$

where $\Phi_{l}^{g}$ is the general solution of the corresponding homogeneous equation

$D(s, \boldsymbol{\xi}) \Phi_{l}^{g}=\rho^{2}\left(s^{2}-b^{2}\right)\left(s^{2}-a^{2}\right) \Phi_{l}^{g}=0$,

and $\Phi_{l}^{p}$ is the particular solution of (3.10), which, in the sense of distributions, is given by

$\Phi_{l}^{p}=-\frac{N_{l j}}{D} K_{j}(\boldsymbol{\xi}) \partial_{s}^{3} \hat{H}(s, \boldsymbol{\xi})=-L_{l j}^{-1} K_{j}(\boldsymbol{\xi}) \partial_{s}^{3} \hat{H}(s, \boldsymbol{\xi})$.

In Eq. (3.12), the coefficient of the unknown $\partial_{s}^{4} \hat{\phi}_{l}$ has zeros at $s= \pm a, \pm b$, and, without loss of generality, the general solution $\Phi_{l}^{g}$ of the homogeneous 
equation (3.12) is written as,

$\Phi_{l}^{g}=\left.\sum_{\text {poles } q_{i}}\left[\frac{N_{l j}(\gamma, \boldsymbol{\xi})}{\partial_{\gamma} D(\gamma, \boldsymbol{\xi})}\right]\right|_{\gamma=q_{i}} K_{j}(\boldsymbol{\xi}) f_{i} \delta\left(s-q_{i}\right)$,

where $f_{i}$, for $i=1,2,3,4$, are undetermined functions, and the poles $q_{i}$ are $q_{1}=a, q_{2}=-a, q_{3}=b$, and $q_{4}=-b$.

From (3.8) and $(3.9), N_{j l}(\gamma, \boldsymbol{\xi})$ and $D(\gamma, \boldsymbol{\xi})$ are even functions of $\gamma$, hence $\partial_{\gamma} D$ is odd in $\gamma$ and Eq. (3.14) is rewritten as

$$
\begin{aligned}
\Phi_{l}^{g}= & {\left[\frac{N_{l j}(a, \boldsymbol{\xi})}{\left.\partial_{\gamma} D(\gamma, \boldsymbol{\xi})\right|_{\gamma=a}}\right] K_{j}\left[f_{1}(a, \boldsymbol{\xi}) \delta(s-a)-f_{2}(a, \boldsymbol{\xi}) \delta(s+a)\right] } \\
& +\left[\frac{N_{l j}(b, \boldsymbol{\xi})}{\left.\partial_{\gamma} D(\gamma, \boldsymbol{\xi})\right|_{\gamma=b}}\right] K_{j}\left[f_{3}(b, \boldsymbol{\xi}) \delta(s-b)-f_{4}(b, \boldsymbol{\xi}) \delta(s+b)\right] .
\end{aligned}
$$

Combining Eqs. (3.13) and (3.15), we have the solution of $\partial_{s}^{4} \hat{\phi_{m}}(s, \boldsymbol{\xi})$

$$
\begin{aligned}
\partial_{s}^{4} \hat{\phi}_{l}(s, \boldsymbol{\xi}) & =\Phi_{l}^{p}+\Phi_{l}^{g}=-L_{l j}^{-1} K_{j}(\boldsymbol{\xi}) \partial_{s}^{3} \hat{H}(s, \boldsymbol{\xi}) \\
& +\left[\frac{N_{l j}(a, \boldsymbol{\xi})}{\left.\partial_{\gamma} D(\gamma, \boldsymbol{\xi})\right|_{\gamma=a}}\right] K_{j}\left[f_{1}(a, \boldsymbol{\xi}) \delta(s-a)-f_{2}(a, \boldsymbol{\xi}) \delta(s+a)\right] \\
& +\left[\frac{N_{l j}(b, \boldsymbol{\xi})}{\left.\partial_{\gamma} D(\gamma, \boldsymbol{\xi})\right|_{\gamma=b}}\right] K_{j}\left[f_{3}(b, \boldsymbol{\xi}) \delta(s-b)-f_{4}(b, \boldsymbol{\xi}) \delta(s+b)\right] .
\end{aligned}
$$

In Eq. (3.16), we need to evaluate the term $L_{l j}^{-1} K_{j}(\boldsymbol{\xi}) \partial_{s}^{3} \hat{H}(s, \boldsymbol{\xi})$, which will follow next. We note that the integrand of $\hat{H}(s, \boldsymbol{\xi})$ defined by $(3.5)$ contains a delta function concentrated on a plane $\left\{\boldsymbol{z} \mid z_{k} \xi_{k}=s\right\}$; such particular type of generalized functions have been discussed in Gel'fand and Shilov (1964). For a fixed unit vector $\boldsymbol{\xi} \in S$ and a real number $s, H\left(1-s_{k}^{2} z_{k}^{2}\right) \delta\left(s-z_{k} \xi_{k}\right)$ represents the intersection of the ellipsoid $\left\{\boldsymbol{z} \mid s_{k}^{2} z_{k}^{2} \leq 1\right\}$ and the plane $\left\{\boldsymbol{z} \mid z_{k} \xi_{k}=s\right\}$. The intersection is defined by a bounded quadratic function of two variables, so that it is an elliptic disc (if it is not empty). Consequently, $\hat{H}(s, \boldsymbol{\xi})$ defined by the integral (3.5) equals the area of the intersection ellipse. And, we have, (see 
Gel'fand and Shilov, 1964)

$\hat{H}(s, \boldsymbol{\xi})=\int_{R^{3}} H\left(1-s_{k}^{2} z_{k}^{2}\right) \delta\left(s-z_{k} \xi_{k}\right) d z=A_{s} H\left(p_{\xi}-|s|\right)$,

where $A_{s}$ is the area of the intersection elliptic disc, which is found to be

$A_{s}=\frac{\pi}{s_{1} s_{2} s_{3} p_{\xi}^{3}}\left(p_{\xi}^{2}-s^{2}\right)$

(see, e.g., Ferguson, 1979), and $p_{\xi}=p_{\xi}(\boldsymbol{\xi})$ is the maximum value of $|s|$ for which the intersection ellipse disc is not empty. In other words, the planes defined by $s=(\boldsymbol{z} \cdot \boldsymbol{\xi})= \pm p_{\xi}$ are tangent to the ellipsoid $\left\{\boldsymbol{z} \mid s_{\kappa}^{2} z_{\kappa}^{2} \leq 1\right\}$. Let us assume the tangent point is $\hat{\boldsymbol{z}}$, then the normal of the ellipsoid at $\hat{\boldsymbol{z}}$ is in the direction of $\boldsymbol{\xi}$, namely,

$\left(\xi_{1}, \xi_{2}, \xi_{3}\right)=\beta\left(s_{1}^{2} \hat{z}_{1}, s_{2}^{2} \hat{z}_{2}, s_{3}^{2} \hat{z}_{3}\right)$

where the undetermined constant $\beta$ of proportionality can be determined by the condition that $\hat{z}$ is on the boundary of the ellipsoid. It then follows that $\beta= \pm\left(\frac{\xi_{i}^{2}}{s_{i}^{2}}\right)^{1 / 2}$

and

$p_{\xi}=|(\hat{\boldsymbol{z}} \cdot \boldsymbol{\xi})|=\left(\frac{\xi_{i}^{2}}{s_{i}^{2}}\right)^{1 / 2}$.

From Eqs. (3.17) and (3.18), we have

$\partial_{s}^{3} \hat{H}(s, \boldsymbol{\xi})=\frac{\pi}{s_{1} s_{2} s_{3} p_{\xi}^{3}} \partial_{s}^{3}\left[\left(p_{\xi}^{2}-s^{2}\right) H\left(p_{\xi}-|s|\right)\right]$.

Recall the basic relation, for $p_{\xi}>0$,

$H\left(p_{\xi}-|s|\right)=H\left(p_{\xi}+s\right) H\left(p_{\xi}-s\right)$,

$\partial_{s}\left[H\left(p_{\xi}-|s|\right)\right]=\delta\left(p_{\xi}+s\right)-\delta\left(p_{\xi}-s\right)$. 
Hence, we have

$$
\begin{gathered}
\partial_{s}^{3}\left[\left(p_{\xi}^{2}-s^{2}\right) H\left(p_{\xi}-|s|\right)\right]=-2\left[\delta\left(p_{\xi}+s\right)-\delta\left(s-p_{\xi}\right)\right] \\
+2 p_{\xi}\left[\delta^{\prime}\left(p_{\xi}+s\right)+\delta^{\prime}\left(s-p_{\xi}\right)\right]
\end{gathered}
$$

and

$$
\begin{gathered}
-L_{l j}^{-1}(s, \boldsymbol{\xi}) \partial_{s}^{3} \hat{H}(s, \boldsymbol{\xi})=\frac{\pi}{s_{1} s_{2} s_{3} p_{\xi}^{3}}\left\{2 L_{l j}^{-1}(s, \boldsymbol{\xi})\left[\delta\left(s+p_{\xi}\right)-\delta\left(s-p_{\xi}\right)\right]\right. \\
\left.-2 p_{\xi} L_{l j}^{-1}(s, \boldsymbol{\xi})\left[\delta^{\prime}\left(p_{\xi}+s\right)+\delta^{\prime}\left(s-p_{\xi}\right)\right]\right\} .
\end{gathered}
$$

Eq. (3.26) is further reduced to

$$
\begin{aligned}
-L_{l j}^{-1}(s, \boldsymbol{\xi}) \partial_{s}^{3} \hat{H}(s, \boldsymbol{\xi}) & =\frac{\pi}{s_{1} s_{2} s_{3} p_{\xi}^{3}}\left\{-2 p_{\xi} L_{l j}^{-1}\left(p_{\xi}, \boldsymbol{\xi}\right)\left[\delta^{\prime}\left(p_{\xi}+s\right)+\delta^{\prime}\left(s-p_{\xi}\right)\right]\right. \\
& \left.+\left[4 \rho p_{\xi}^{2} L_{l j}^{-2}\left(p_{\xi}, \boldsymbol{\xi}\right)+2 L_{l j}^{-1}\left(p_{\xi}, \boldsymbol{\xi}\right)\right]\left[\delta\left(s+p_{\xi}\right)-\delta\left(s-p_{\xi}\right)\right]\right\},
\end{aligned}
$$

where we have used the basic identity of the delta function

$f(x) \delta^{\prime}(x)=-f^{\prime}(0) \delta(x)+f(0) \delta^{\prime}(x)$,

and the relation

$\partial_{s}\left[L_{l j}^{-1}(s, \boldsymbol{\xi})\right]=-L_{l i}^{-2}(s, \boldsymbol{\xi}) \partial_{s}\left(L_{i j}(s, \boldsymbol{\xi})\right)=-2 \rho s L_{l j}^{-2}(s, \boldsymbol{\xi})$.

By using (3.23) and

$\delta\left(p_{\xi}-|s|\right)=\delta\left(s+p_{\xi}\right)+\delta\left(s-p_{\xi}\right)$,

we may further simplify Eq. (3.27) to

$$
\begin{gathered}
-L_{l j}^{-1}(s, \boldsymbol{\xi}) \partial_{s}^{3} \hat{H}(s, \boldsymbol{\xi})=\frac{\pi}{s_{1} s_{2} s_{3} p_{\xi}^{3}}\left\{-2 p_{\xi} L_{l j}^{-1}(p, \boldsymbol{\xi}) \partial_{s}\left[\delta\left(p_{\xi}-|s|\right)\right]\right. \\
\left.+\left[4 \rho p_{\xi}^{2} L_{l j}^{-2}\left(p_{\xi}, \boldsymbol{\xi}\right)+2 L_{l j}^{-1}\left(p_{\xi}, \boldsymbol{\xi}\right)\right] \partial_{s}\left[H\left(p_{\xi}-|s|\right)\right]\right\} .
\end{gathered}
$$


Substituting (3.31) into (3.16), we obtain

$$
\begin{aligned}
\partial_{s}^{4} & \hat{\phi}_{l}(s, \boldsymbol{\xi})=\frac{\pi}{s_{1} s_{2} s_{3} p_{\xi}^{3}}\left\{-2 p_{\xi} L_{l j}^{-1}\left(p_{\xi}, \boldsymbol{\xi}\right) K_{j}(\boldsymbol{\xi}) \partial_{s}\left[\delta\left(p_{\xi}-|s|\right)\right]\right. \\
& \left.+\left[4 \rho p_{\xi}^{2} L_{l j}^{-2}\left(p_{\xi}, \boldsymbol{\xi}\right)+2 L_{l j}^{-1}\left(p_{\xi}, \boldsymbol{\xi}\right)\right] K_{j}(\boldsymbol{\xi}) \partial_{s}\left[H\left(p_{\xi}-|s|\right)\right]\right\} \\
& +\left[\frac{N_{l j}(a, \boldsymbol{\xi})}{\left.\partial_{\gamma} D(\gamma, \boldsymbol{\xi})\right|_{\gamma=a}}\right] K_{j}\left[f_{1}(a, \boldsymbol{\xi}) \delta(s-a)-f_{2}(a, \boldsymbol{\xi}) \delta(s+a)\right] \\
& +\left[\frac{N_{l j}(b, \boldsymbol{\xi})}{\left.\partial_{\gamma} D(\gamma, \boldsymbol{\xi})\right|_{\gamma=b}}\right] K_{j}\left[f_{3}(b, \boldsymbol{\xi}) \delta(s-b)-f_{4}(b, \boldsymbol{\xi}) \delta(s+b)\right] .
\end{aligned}
$$

In order to derive the solution $\phi_{l}(\boldsymbol{z})$ from the inverse Radon transform (3.2), by double integration of Eq. (3.32) with respect to $s$, we obtain $\partial_{s}^{2} \hat{\phi}_{l}(s, \boldsymbol{\xi})$

$$
\begin{aligned}
& \partial_{s}^{2} \hat{\phi}_{l}(s, \boldsymbol{\xi})=\frac{\pi}{s_{1} s_{2} s_{3}}\left\{2 p_{\xi}^{-3} L_{l j}^{-1}\left(p_{\xi}, \boldsymbol{\xi}\right) K_{j}(\boldsymbol{\xi})\left[s H\left(p_{\xi}-|s|\right)\right]\right. \\
& \left.\quad+2 \rho p_{\xi}^{-1} L_{l j}^{-2}\left(p_{\xi}, \boldsymbol{\xi}\right) K_{j}(\boldsymbol{\xi})\left[\left(p_{\xi}+s\right) \operatorname{sgn}\left(p_{\xi}+s\right)-\left(p_{\xi}-s\right) \operatorname{sgn}\left(p_{\xi}-s\right)\right]\right\} \\
& +\left[\frac{N_{l j}(a, \boldsymbol{\xi})}{\left.\partial_{\gamma} D(\gamma, \boldsymbol{\xi})\right|_{\gamma=a}}\right] K_{j}\left[f_{1}(a, \boldsymbol{\xi})(s-a) \operatorname{sgn}(s-a)-f_{2}(a, \boldsymbol{\xi})(s+a) \operatorname{sgn}(s+a)\right] \\
& +\left[\frac{N_{l j}(b, \boldsymbol{\xi})}{\left.\partial_{\gamma} D(\gamma, \boldsymbol{\xi})\right|_{\gamma=b}}\right] K_{j}\left[f_{3}(b, \boldsymbol{\xi})(s-b) \operatorname{sgn}(s-b)-f_{4}(b, \boldsymbol{\xi})(s+b) \operatorname{sgn}(s+b)\right] \\
& +g_{1}(\boldsymbol{\xi}) s+g_{2}(\boldsymbol{\xi}),
\end{aligned}
$$

where we have used the relation $\partial_{s}[\operatorname{sgn}(s \pm x)]=2 \delta(s \pm x)$ and

$H\left(p_{\xi}-|s|\right)=\frac{1}{2}\left[\operatorname{sgn}\left(p_{\xi}+s\right)+\operatorname{sgn}\left(p_{\xi}-s\right)\right]$,

and $g_{k}, k=1,2$, are undetermined functions.

By the inverse Radon transform (3.2), the solution of the system of governing equation (2.24) is obtained through a surface integration of (3.33) over the unit sphere,

$$
\begin{aligned}
& \phi_{l}(\boldsymbol{z})=\frac{-1}{8 \pi^{2}} \int_{|\boldsymbol{\xi}|=1}\left\{\frac { \pi } { s _ { 1 } s _ { 2 } s _ { 3 } } \left[2 p_{\xi}^{-3} L_{l j}^{-1}\left(p_{\xi}, \boldsymbol{\xi}\right) K_{j}(\boldsymbol{\xi})\left[s H\left(p_{\xi}-|s|\right)\right]\right.\right. \\
& \left.+2 \rho p_{\xi}^{-1} L_{l j}^{-2}\left(p_{\xi}, \boldsymbol{\xi}\right) K_{j}(\boldsymbol{\xi})\left[\left(p_{\xi}+s\right) \operatorname{sgn}\left(p_{\xi}+s\right)-\left(p_{\xi}-s\right) \operatorname{sgn}\left(p_{\xi}-s\right)\right]\right] \\
& +\left[\frac{N_{l j}(a, \boldsymbol{\xi})}{\left.\partial_{\gamma} D(\gamma, \boldsymbol{\xi})\right|_{\gamma=a}}\right] K_{j}\left[f_{1}(a, \boldsymbol{\xi})(s-a) \operatorname{sgn}(s-a)-f_{2}(a, \boldsymbol{\xi})(s+a) \operatorname{sgn}(s+a)\right]
\end{aligned}
$$




$$
\begin{aligned}
& \left.+\left[\frac{N_{l j}(b, \boldsymbol{\xi})}{\left.\partial_{\gamma} D(\gamma, \boldsymbol{\xi})\right|_{\gamma=b}}\right] K_{j}\left[f_{3}(b, \boldsymbol{\xi})(s-b) \operatorname{sgn}(s-b)-f_{4}(b, \boldsymbol{\xi})(s+b) \operatorname{sgn}(s+b)\right]\right\}\left.\right|_{s=(\boldsymbol{z} \cdot \boldsymbol{\xi})} d S \\
& -\frac{1}{8 \pi^{2}} \int_{|\boldsymbol{\xi}|=1}\left[g_{1}(\boldsymbol{\xi})(\boldsymbol{z} \cdot \boldsymbol{\xi})+g_{2}(\boldsymbol{\xi})\right] d S
\end{aligned}
$$

where $s=(\boldsymbol{z} \cdot \boldsymbol{\xi})$. Hence, we have the solution of the displacement

$$
\begin{aligned}
& u_{l}(\boldsymbol{x}, t)=\frac{-1}{8 \pi^{2}} \int_{|\boldsymbol{\xi}|=1}\left\{\frac { \pi } { s _ { 1 } s _ { 2 } s _ { 3 } } \left[2 p_{\xi}^{-3} L_{l j}^{-1}\left(p_{\xi}, \boldsymbol{\xi}\right) K_{j}(\boldsymbol{\xi})\left[(\boldsymbol{x} \cdot \boldsymbol{\xi}) H\left(p_{\xi} t-|\boldsymbol{x} \cdot \boldsymbol{\xi}|\right)\right]\right.\right. \\
& \left.+2 \rho p_{\xi}^{-1} L_{l j}^{-2}\left(p_{\xi}, \boldsymbol{\xi}\right) K_{j}(\boldsymbol{\xi})\left[\left(p_{\xi} t+\boldsymbol{x} \cdot \boldsymbol{\xi}\right) \operatorname{sgn}\left(p_{\xi} t+\boldsymbol{x} \cdot \boldsymbol{\xi}\right)-\left(p_{\xi} t-\boldsymbol{x} \cdot \boldsymbol{\xi}\right) \operatorname{sgn}\left(p_{\xi} t-\boldsymbol{x} \cdot \boldsymbol{\xi}\right)\right]\right] \\
& +\left[\frac{N_{l j}(a, \boldsymbol{\xi})}{\left.\partial_{\gamma} D(\gamma, \boldsymbol{\xi})\right|_{\gamma=a}}\right] K_{j}\left[f_{1}(a, \boldsymbol{\xi})(\boldsymbol{x} \cdot \boldsymbol{\xi}-a t) \operatorname{sgn}(\boldsymbol{x} \cdot \boldsymbol{\xi}-a t)-f_{2}(a, \boldsymbol{\xi})(\boldsymbol{x} \cdot \boldsymbol{\xi}+a t) \operatorname{sgn}(\boldsymbol{x} \cdot \boldsymbol{\xi}+a t)\right] \\
& \left.+\left[\frac{N_{l j}(b, \boldsymbol{\xi})}{\left.\partial_{\gamma} D(\gamma, \boldsymbol{\xi})\right|_{\gamma=b}}\right] K_{j}\left[f_{3}(b, \boldsymbol{\xi})(\boldsymbol{x} \cdot \boldsymbol{\xi}-b t) \operatorname{sgn}(\boldsymbol{x} \cdot \boldsymbol{\xi}-b t)-f_{4}(b, \boldsymbol{\xi})(\boldsymbol{x} \cdot \boldsymbol{\xi}+b t) \operatorname{sgn}(\boldsymbol{x} \cdot \boldsymbol{\xi}+b t)\right]\right\} d S \\
& -\frac{1}{8 \pi^{2}} \int_{|\boldsymbol{\xi}|=1}\left[g_{1}(\boldsymbol{\xi})(\boldsymbol{x} \cdot \boldsymbol{\xi})+t g_{2}(\boldsymbol{\xi})\right] d S .
\end{aligned}
$$

We will apply the initial conditions (2.4) and (2.5) to find the undetermined functions $f_{i}, i=1,2,3,4$, and $g_{k}, k=1,2$, and with these determined functions, we prove that the boundary condition (??) is also satisfied. Then, by the uniqueness theorem for an unbounded domain (Wheeler and Sternberg, 1968), the obtained solution is the unique solution for the governing equations (2.3) with the initial and boundary conditions (2.4), (2.5), and (??).

\subsection{The application of the initial conditions}

From Eq. (3.36), we have the expression for the displacement at $t=0$,

$$
\begin{aligned}
& u_{l}(\boldsymbol{x}, 0)=\frac{-1}{8 \pi^{2}} \int_{|\boldsymbol{\xi}|=1}\left\{\left[\frac{N_{l j}(a, \boldsymbol{\xi})}{\left.\partial_{\gamma} D(\gamma, \boldsymbol{\xi})\right|_{\gamma=a}}\right] K_{j}\left[f_{1}(a, \boldsymbol{\xi})-f_{2}(a, \boldsymbol{\xi})\right](\boldsymbol{x} \cdot \boldsymbol{\xi}) \operatorname{sgn}(\boldsymbol{x} \cdot \boldsymbol{\xi})\right. \\
& \left.+\left[\frac{N_{l j}(b, \boldsymbol{\xi})}{\left.\partial_{\gamma} D(\gamma, \boldsymbol{\xi})\right|_{\gamma=b}}\right] K_{j}\left[f_{3}(b, \boldsymbol{\xi})-f_{4}(b, \boldsymbol{\xi})\right](\boldsymbol{x} \cdot \boldsymbol{\xi}) \operatorname{sgn}(\boldsymbol{x} \cdot \boldsymbol{\xi})\right\} d S \\
& -\frac{1}{8 \pi^{2}} \int_{|\boldsymbol{\xi}|=1} g_{1}(\boldsymbol{\xi})(\boldsymbol{x} \cdot \boldsymbol{\xi}) d S .
\end{aligned}
$$


To satisfy the initial condition $\boldsymbol{u}(\boldsymbol{x}, 0)=0$ for all $\boldsymbol{x}$, since on the right hand side of Eq. (3.37), the first term is an even function of $\boldsymbol{x}$, and the second term is an odd function of $\boldsymbol{x}$, follows that both terms are zero. Hence, we have

$$
\int_{|\boldsymbol{\xi}|=1} g_{1}(\boldsymbol{\xi})(\boldsymbol{x} \cdot \boldsymbol{\xi}) d S=0 .
$$

From Eqs. (3.4), (3.8), and (3.9), in the first term of the right hand side of Eq. (3.37), the following factors

$\left[\frac{N_{l j}(a, \boldsymbol{\xi})}{\left.\partial_{\gamma} D(\gamma, \boldsymbol{\xi})\right|_{\gamma=a}}\right] K_{j}, \quad$ and $\quad\left[\frac{N_{l j}(b, \boldsymbol{\xi})}{\left.\partial_{\gamma} D(\gamma, \boldsymbol{\xi})\right|_{\gamma=b}}\right] K_{j}$,

are odd functions of $\boldsymbol{\xi}$. To satisfy the first term of the right hand side of Eq. (3.37) equal to zero, we have $f_{1}-f_{2}$ and $f_{3}-f_{4}$ are even functions of $\boldsymbol{\xi}$.

We notice that in the expression (3.36) of $u_{l}(\boldsymbol{x}, t)$, we may rewrite the factor in the integrand $f_{1}(\boldsymbol{x} \cdot \boldsymbol{\xi}-a t) \operatorname{sgn}(\boldsymbol{x} \cdot \boldsymbol{\xi}-a t)-f_{2}(\boldsymbol{x} \cdot \boldsymbol{\xi}+a t) \operatorname{sgn}(\boldsymbol{x} \cdot \boldsymbol{\xi}+a t)$ in the following way

$$
\begin{aligned}
& f_{1}(\boldsymbol{x} \cdot \boldsymbol{\xi}-a t) \operatorname{sgn}(\boldsymbol{x} \cdot \boldsymbol{\xi}-a t)-f_{2}(\boldsymbol{x} \cdot \boldsymbol{\xi}+a t) \operatorname{sgn}(\boldsymbol{x} \cdot \boldsymbol{\xi}+a t)= \\
& \frac{1}{2}\left(f_{1}+f_{2}\right)[(\boldsymbol{x} \cdot \boldsymbol{\xi}-a t) \operatorname{sgn}(\boldsymbol{x} \cdot \boldsymbol{\xi}-a t)-(\boldsymbol{x} \cdot \boldsymbol{\xi}+a t) \operatorname{sgn}(\boldsymbol{x} \cdot \boldsymbol{\xi}+a t)] \\
& +\frac{1}{2}\left(f_{1}-f_{2}\right)[(\boldsymbol{x} \cdot \boldsymbol{\xi}-a t) \operatorname{sgn}(\boldsymbol{x} \cdot \boldsymbol{\xi}-a t)+(\boldsymbol{x} \cdot \boldsymbol{\xi}+a t) \operatorname{sgn}(\boldsymbol{x} \cdot \boldsymbol{\xi}+a t)],
\end{aligned}
$$

where we notice that the $\left(f_{1}-f_{2}\right)[(\boldsymbol{x} \cdot \boldsymbol{\xi}-a t) \operatorname{sgn}(\boldsymbol{x} \cdot \boldsymbol{\xi}-a t)+(\boldsymbol{x} \cdot \boldsymbol{\xi}+a t) \operatorname{sgn}(\boldsymbol{x}$. $\boldsymbol{\xi}+a t)]$ term in this expression is an even function of $\boldsymbol{\xi}$. By substituting the expression (3.40) into the integral in the first term of the right hand side of Eq. (3.36), as an integral of an odd function over a symmetry integral domain (the whole unit sphere), the integral of the following term is zero

$$
\begin{aligned}
& {\left[\frac{N_{l j}(a, \boldsymbol{\xi})}{\left.\partial_{\gamma} D(\gamma, \boldsymbol{\xi})\right|_{\gamma=a}}\right] K_{j}} \\
& \frac{\left(f_{1}-f_{2}\right)}{2}[(\boldsymbol{x} \cdot \boldsymbol{\xi}-a t) \operatorname{sgn}(\boldsymbol{x} \cdot \boldsymbol{\xi}-a t)+(\boldsymbol{x} \cdot \boldsymbol{\xi}+a t) \operatorname{sgn}(\boldsymbol{x} \cdot \boldsymbol{\xi}+a t)] .
\end{aligned}
$$

The same conclusion holds for the term invoving $f_{3}$ and $f_{4}$ in Eq. (3.36). Hence, the expression (3.36) for the displacement is reduced to 


$$
\begin{aligned}
& u_{l}(\boldsymbol{x}, t)=\frac{-1}{8 \pi^{2}} \int_{|\boldsymbol{\xi}|=1}\left\{\frac { \pi } { s _ { 1 } s _ { 2 } s _ { 3 } } \left[2 p_{\xi}^{-3} L_{l j}^{-1}\left(p_{\xi}, \boldsymbol{\xi}\right) K_{j}(\boldsymbol{\xi})\left[(\boldsymbol{x} \cdot \boldsymbol{\xi}) H\left(p_{\xi} t-|\boldsymbol{x} \cdot \boldsymbol{\xi}|\right)\right]\right.\right. \\
& \left.+2 \rho p_{\xi}^{-1} L_{l j}^{-2}\left(p_{\xi}, \boldsymbol{\xi}\right) K_{j}(\boldsymbol{\xi})\left[\left(p_{\xi} t+\boldsymbol{x} \cdot \boldsymbol{\xi}\right) \operatorname{sgn}\left(p_{\xi} t+\boldsymbol{x} \cdot \boldsymbol{\xi}\right)-\left(p_{\xi} t-\boldsymbol{x} \cdot \boldsymbol{\xi}\right) \operatorname{sgn}\left(p_{\xi} t-\boldsymbol{x} \cdot \boldsymbol{\xi}\right)\right]\right] \\
& +\left[\frac{N_{l j}(a, \boldsymbol{\xi})}{\left.\partial_{\gamma} D(\gamma, \boldsymbol{\xi})\right|_{\gamma=a}}\right] K_{j} \frac{\left(f_{1}+f_{2}\right)}{2}[(\boldsymbol{x} \cdot \boldsymbol{\xi}-a t) \operatorname{sgn}(\boldsymbol{x} \cdot \boldsymbol{\xi}-a t)-(\boldsymbol{x} \cdot \boldsymbol{\xi}+a t) \operatorname{sgn}(\boldsymbol{x} \cdot \boldsymbol{\xi}+a t)] \\
& \left.+\left[\frac{N_{l j}(b, \boldsymbol{\xi})}{\left.\partial_{\gamma} D(\gamma, \boldsymbol{\xi})\right|_{\gamma=b}}\right] K_{j} \frac{\left(f_{3}+f_{4}\right)}{2}[(\boldsymbol{x} \cdot \boldsymbol{\xi}-b t) \operatorname{sgn}(\boldsymbol{x} \cdot \boldsymbol{\xi}-b t)-(\boldsymbol{x} \cdot \boldsymbol{\xi}+b t) \operatorname{sgn}(\boldsymbol{x} \cdot \boldsymbol{\xi}+b t)]\right\} d S \\
& -\frac{1}{8 \pi^{2}} \int_{|\boldsymbol{\xi}|=1} t g_{2}(\boldsymbol{\xi}) d S .
\end{aligned}
$$

From Eq. (3.42), we have the particle velocity at $t=0$,

$$
\begin{aligned}
& \frac{\partial u_{l}(\boldsymbol{x}, 0)}{\partial t}=\frac{-1}{8 \pi^{2}} \int_{|\boldsymbol{\xi}|=1}\left\{\frac{4 \pi \rho}{s_{1} s_{2} s_{3}} L_{l j}^{-2}\left(p_{\xi}, \boldsymbol{\xi}\right) K_{j}(\boldsymbol{\xi}) \operatorname{sgn}(\boldsymbol{x} \cdot \boldsymbol{\xi})\right. \\
& \left.-a\left[\frac{N_{l j}(a, \boldsymbol{\xi})}{\left.\partial_{\gamma} D(\gamma, \boldsymbol{\xi})\right|_{\gamma=a}}\right] K_{j}(\boldsymbol{\xi})\left(f_{1}+f_{2}\right) \operatorname{sgn}(\boldsymbol{x} \cdot \boldsymbol{\xi})-b\left[\frac{N_{l j}(b, \boldsymbol{\xi})}{\left.\partial_{\gamma} D(\gamma, \boldsymbol{\xi})\right|_{\gamma=b}}\right] K_{j}(\boldsymbol{\xi})\left(f_{3}+f_{4}\right) \operatorname{sgn}(\boldsymbol{x} \cdot \boldsymbol{\xi})\right\} d S \\
& -\frac{1}{8 \pi^{2}} \int_{|\boldsymbol{\xi}|=1} g_{2}(\boldsymbol{\xi}) d S .
\end{aligned}
$$

Since the initial condition $(2.5) \partial u_{l} / \partial t(\boldsymbol{x}, 0)=0$ valid for all $\boldsymbol{x}$, and the first term on the right hand side of Eq. (3.43) is an odd function of $\boldsymbol{x}$, while the second term on the right hand side of Eq. (3.43) is independent of $\boldsymbol{x}$, hence we have

$$
\int_{|\boldsymbol{\xi}|=1} g_{2}(\boldsymbol{\xi}) d S=0
$$

and both terms in (3.43) are zero identically.

As for the first integral in the expression of $\partial u_{l} / \partial t(\boldsymbol{x}, 0)$, notice that for an isotropic medium that is solved here, for $|\boldsymbol{\xi}|=1$, we have

$$
L_{l j}^{-1}\left(p_{\xi}, \boldsymbol{\xi}\right)=\frac{\rho\left[\left(p_{\xi}^{2}-a^{2}\right) \delta_{l j}+\left(a^{2}-b^{2}\right) \xi_{l} \xi_{j}\right]}{\rho^{2}\left(p_{\xi}^{2}-a^{2}\right)\left(p_{\xi}^{2}-b^{2}\right)}=\frac{1}{\rho}\left[\frac{\delta_{l j}-\xi_{l} \xi_{j}}{\left(p_{\xi}^{2}-b^{2}\right)}+\frac{\xi_{l} \xi_{j}}{\left(p_{\xi}^{2}-a^{2}\right)}\right]
$$


$L_{l j}^{-2}\left(p_{\xi}, \boldsymbol{\xi}\right)=L_{l k}^{-1} L_{k j}^{-1}=\frac{1}{\rho^{2}}\left[\frac{\delta_{l j}-\xi_{l} \xi_{j}}{\left(p_{\xi}^{2}-b^{2}\right)^{2}}+\frac{\xi_{l} \xi_{j}}{\left(p_{\xi}^{2}-a^{2}\right)^{2}}\right]$,

and

$\frac{N_{l j}(a, \boldsymbol{\xi})}{\left.\partial_{\gamma} D(\gamma, \boldsymbol{\xi})\right|_{\gamma=a}}=\frac{\rho\left(a^{2}-b^{2}\right) \xi_{l} \xi_{j}}{2 a \rho^{2}\left(a^{2}-b^{2}\right)}=\frac{\xi_{l} \xi_{j}}{2 a \rho}$,

$\frac{N_{l j}(b, \boldsymbol{\xi})}{\left.\partial_{\gamma} D(\gamma, \boldsymbol{\xi})\right|_{\gamma=b}}=\frac{\rho\left(b^{2}-a^{2}\right)\left[\delta_{l j}-\xi_{l} \xi_{j}\right]}{2 b \rho^{2}\left(b^{2}-a^{2}\right)}=\frac{\delta_{l j}-\xi_{l} \xi_{j}}{2 b \rho}$.

If we define $\boldsymbol{\zeta}=\boldsymbol{x} /|\boldsymbol{x}|$ as the direction of $\boldsymbol{x}$, then Eq. (3.43) is reduced to

$$
\begin{gathered}
\frac{\partial u_{l}(\boldsymbol{x}, 0)}{\partial t}=\frac{-1}{8 \pi^{2}} \int_{|\boldsymbol{\xi}|=1}\left\{\frac{4 \pi}{\rho s_{1} s_{2} s_{3}}\left[\frac{\xi_{l} \xi_{j}}{\left(p_{\boldsymbol{\xi}}^{2}-a^{2}\right)^{2}}+\frac{\delta_{l j}-\xi_{l} \xi_{j}}{\left(p_{\boldsymbol{\xi}}^{2}-b^{2}\right)^{2}}\right] \operatorname{sgn}(\boldsymbol{\zeta} \cdot \boldsymbol{\xi})\right. \\
\left.-\frac{1}{2 \rho}\left[\xi_{l} \xi_{j}\left(f_{1}+f_{2}\right)+\left(\delta_{l j}-\xi_{l} \xi_{j}\right)\left(f_{3}+f_{4}\right)\right] \operatorname{sgn}(\boldsymbol{\zeta} \cdot \boldsymbol{\xi})\right\} K_{j}(\boldsymbol{\xi}) d S .
\end{gathered}
$$

So that for the only undetermined coefficients $f_{1}+f_{2}$ and $f_{3}+f_{4}$, in order to satisfy the initial condition (2.5), we have

$$
\begin{aligned}
f_{1}+f_{2} & =\frac{8 \pi}{s_{1} s_{2} s_{3}\left(a^{2}-p_{\xi}^{2}\right)^{2}}, \\
f_{3}+f_{4} & =\frac{8 \pi}{s_{1} s_{2} s_{3}\left(a^{2}-p_{\xi}^{2}\right)^{2}} .
\end{aligned}
$$

From Eq. (3.42) with the choice of the undetermined functions by (3.44), (3.50), and (3.51), and by using Eqs. (3.45) and (3.46) for the operators $L^{-1}$ and $L^{-2}$, we have the expression for the displacement

$$
\begin{aligned}
& u_{l}(\boldsymbol{x}, t)=\frac{-1}{4 \pi s_{1} s_{2} s_{3}} \int_{|\boldsymbol{\xi}|=1}\left\{\frac{1}{\rho p_{\xi}^{3}}\left[\frac{\xi_{\xi} \xi_{j}}{p_{\xi}^{2}-a^{2}}+\frac{\delta_{l j}-\xi_{l} \xi_{j}}{p_{\xi}^{2}-b^{2}}\right] K_{j}(\boldsymbol{\xi})\left[(\boldsymbol{x} \cdot \boldsymbol{\xi}) H\left(p_{\xi} t-|\boldsymbol{x} \cdot \boldsymbol{\xi}|\right)\right]\right. \\
& +\frac{1}{\rho p_{\xi}}\left[\frac{\xi_{l} \xi_{j}}{\left(p_{\xi}^{2}-a^{2}\right)^{2}}+\frac{\delta_{l j}-\xi_{l} \xi_{j}}{\left(p_{\xi}^{2}-b^{2}\right)^{2}}\right] K_{j}(\boldsymbol{\xi})\left[\left(p_{\xi} t+\boldsymbol{x} \cdot \boldsymbol{\xi}\right) \operatorname{sgn}\left(p_{\xi} t+\boldsymbol{x} \cdot \boldsymbol{\xi}\right)\right. \\
& +\frac{1}{4 \pi s_{1} s_{2} s_{3}} \int_{|\boldsymbol{\xi}|=1}\left\{\frac{\xi_{l} \xi_{j}}{a \rho\left(a^{2}-p_{\xi}^{2}\right)^{2}} K_{j}(\boldsymbol{\xi})[(\boldsymbol{x} \cdot \boldsymbol{\xi}+a t) \operatorname{sgn}(\boldsymbol{x} \cdot \boldsymbol{\xi}+a t)\right.
\end{aligned}
$$




$$
\begin{aligned}
& \quad-(\boldsymbol{x} \cdot \boldsymbol{\xi}-a t) \operatorname{sgn}(\boldsymbol{x} \cdot \boldsymbol{\xi}-a t)] \\
& \left.+\frac{\delta_{l j}-\xi_{l} \xi_{j}}{b \rho\left(b^{2}-p_{\xi}^{2}\right)^{2}} K_{j}(\boldsymbol{\xi})[(\boldsymbol{x} \cdot \boldsymbol{\xi}+b t) \operatorname{sgn}(\boldsymbol{x} \cdot \boldsymbol{\xi}+b t)-(\boldsymbol{x} \cdot \boldsymbol{\xi}-b t) \operatorname{sgn}(\boldsymbol{x} \cdot \boldsymbol{\xi}-b t)]\right\} d S
\end{aligned}
$$

The form of the expression Eq. (3.52) is in agreement with Eqs. (3.19) and (3.20) of Burridge and Willis (1969) for a two-dimensional elliptical selfsimilarly expanding crack.

3.3 The solution for the displacement $u_{l}(\boldsymbol{x}, t)$ of a self-similarly subsonically expanding ellipsoidal inclusion

In Eq. (3.52), the terms involving the pairs $\operatorname{sgn}(\boldsymbol{z} \cdot \boldsymbol{\xi}+\star)$ and $\operatorname{sgn}(\boldsymbol{z} \cdot \boldsymbol{\xi}-\star)$, due to symmetries, are combined to one, we rewrite the solution for displacement filed as

$$
\begin{aligned}
u_{l}(\boldsymbol{x}, t)= & \frac{-1}{4 \pi s_{1} s_{2} s_{3}} \int \frac{1}{|\boldsymbol{\xi}|=1}\left[\frac{\xi_{l} \xi_{j}}{\rho p_{\xi}^{3}}+\frac{\delta_{l j}-\xi_{l} \xi_{j}}{p_{\xi}^{2}-a^{2}}\right] K_{j}(\boldsymbol{\xi})\left[(\boldsymbol{x} \cdot \boldsymbol{\xi}) H\left(p_{\xi} t-|\boldsymbol{x} \cdot \boldsymbol{\xi}|\right)\right] \\
& \left.+\frac{2}{\rho p_{\xi}}\left[\frac{\xi_{l} \xi_{j}}{\left(p_{\xi}^{2}-a^{2}\right)^{2}}+\frac{\delta_{l j}-\xi_{l} \xi_{j}}{\left(p_{\xi}^{2}-b^{2}\right)^{2}}\right] K_{j}(\boldsymbol{\xi})\left[\left(p_{\xi} t+\boldsymbol{x} \cdot \boldsymbol{\xi}\right) \operatorname{sgn}\left(p_{\xi} t+\boldsymbol{x} \cdot \boldsymbol{\xi}\right)\right]\right\} d S \\
+ & \frac{1}{2 \pi s_{1} s_{2} s_{3}} \int\left\{\frac{\xi_{l} \xi_{j}}{a \rho\left(a^{2}-p_{\xi}^{2}\right)^{2}} K_{j}(\boldsymbol{\xi})[(\boldsymbol{x} \cdot \boldsymbol{\xi}+a t) \operatorname{sgn}(\boldsymbol{x} \cdot \boldsymbol{\xi}+a t)]\right. \\
& \left.+\frac{\delta_{l j}-\xi_{l} \xi_{j}}{b \rho\left(b^{2}-p_{\xi}^{2}\right)^{2}} K_{j}(\boldsymbol{\xi})[(\boldsymbol{x} \cdot \boldsymbol{\xi}+b t) \operatorname{sgn}(\boldsymbol{x} \cdot \boldsymbol{\xi}+b t)]\right\} d S
\end{aligned}
$$

where $K_{j}$ is defined in (2.8), and, for an isotropic medium, has the expression

$K_{j}(\boldsymbol{\xi})=C_{j k l m} \epsilon_{l m}^{*} \xi_{k}=\lambda \delta_{j k} \xi_{k} \epsilon_{\kappa \kappa}^{*}+\mu\left(\epsilon_{j k}^{*}+\epsilon_{k j}^{*}\right) \xi_{k}$

3.4 Solution for the displacement gradient $\partial u_{l} / \partial x_{m}(\boldsymbol{x}, t)$ of a self-similarly subsonically expanding ellipsoidal inclusion

From Eq. (3.53) for the displacement, we obtain the displacement gradient 


$$
\begin{gathered}
\frac{\partial u_{l}}{\partial x_{m}}(\boldsymbol{x}, t)=\frac{-1}{4 \pi s_{1} s_{2} s_{3}} \int_{|\boldsymbol{\xi}|=1}\left\{\frac{2 \xi_{m}}{\rho p_{\xi}}\left[\frac{\xi_{l} \xi_{j}}{\left(p_{\xi}^{2}-a^{2}\right)^{2}}+\frac{\delta_{l j}-\xi_{l} \xi_{j}}{\left(p_{\xi}^{2}-b^{2}\right)^{2}}\right] K_{j}(\boldsymbol{\xi}) \operatorname{sgn}\left(p_{\xi} t+\boldsymbol{x} \cdot \boldsymbol{\xi}\right)\right. \\
\left.+\frac{\xi_{m}}{\rho p_{\xi}^{3}}\left[\frac{\xi_{l} \xi_{j}}{p_{\xi}^{2}-a^{2}}+\frac{\delta_{l j}-\xi_{l} \xi_{j}}{p_{\xi}^{2}-b^{2}}\right] K_{j}(\boldsymbol{\xi})\left[H\left(p_{\xi} t-|\boldsymbol{x} \cdot \boldsymbol{\xi}|\right)-p_{\xi} t \delta\left(p_{\xi} t-|\boldsymbol{x} \cdot \boldsymbol{\xi}|\right)\right]\right\} d S \\
+\frac{1}{2 \pi s_{1} s_{2} s_{3}} \int_{|\boldsymbol{\xi}|=1}\left\{\frac{\xi_{m} \xi_{l} \xi_{j}}{a \rho\left(a^{2}-p_{\xi}^{2}\right)^{2}} K_{j}(\boldsymbol{\xi}) \operatorname{sgn}(\boldsymbol{x} \cdot \boldsymbol{\xi}+a t)\right. \\
\left.+\frac{\xi_{m}\left(\delta_{l j}-\xi_{l} \xi_{j}\right)}{b \rho\left(b^{2}-p_{\xi}^{2}\right)^{2}} K_{j}(\boldsymbol{\xi}) \operatorname{sgn}(\boldsymbol{x} \cdot \boldsymbol{\xi}+b t)\right\} d S
\end{gathered}
$$

where we have used (3.24) and (3.30) to derive the relation

$\frac{\partial}{\partial x_{m}}\left[(\boldsymbol{x} \cdot \boldsymbol{\xi}) H\left(p_{\xi} t-|\boldsymbol{x} \cdot \boldsymbol{\xi}|\right)\right]=\xi_{m}\left[H\left(p_{\xi} t-|\boldsymbol{x} \cdot \boldsymbol{\xi}|\right)-p_{\xi} t \delta\left(p_{\xi} t-|\boldsymbol{x} \cdot \boldsymbol{\xi}|\right)\right]$,

and also again used the identity, for a real number $x$,

$\partial_{x}[\operatorname{sgn}(x)]=2 \delta(x)$.

In view of the symmetry of the integrands in $\boldsymbol{\xi}$ and the relation (3.34), the sgn functions in the second integral of Eq. (3.55) are changed to step functions. Combining the like terms, we rewrite Eq. (3.55) as

$$
\begin{gathered}
\frac{\partial u_{l}}{\partial x_{m}}(\boldsymbol{x}, t)=\frac{-1}{4 \pi s_{1} s_{2} s_{3}} \int_{|\boldsymbol{\xi}|=1}\left\{\frac{t \xi_{m}}{\rho p_{\xi}^{2}}\left[\frac{\xi_{l} \xi_{j}}{a^{2}-p_{\xi}^{2}}+\frac{\delta_{l j}-\xi_{l} \xi_{j}}{b^{2}-p_{\xi}^{2}}\right] K_{j}(\boldsymbol{\xi}) \delta\left(p_{\xi} t-|\boldsymbol{x} \cdot \boldsymbol{\xi}|\right)\right. \\
\left.+\frac{\xi_{m}}{\rho p_{\xi}^{3}}\left[\frac{\xi_{l} \xi_{j}\left(3 p_{\xi}^{2}-a^{2}\right)}{\left(p_{\xi}^{2}-a^{2}\right)^{2}}+\frac{\left(\delta_{l j}-\xi_{l} \xi_{j}\right)\left(3 p_{\xi}^{2}-b^{2}\right)}{\left(p_{\xi}^{2}-b^{2}\right)^{2}}\right] K_{j}(\boldsymbol{\xi}) H\left(p_{\xi} t-|\boldsymbol{x} \cdot \boldsymbol{\xi}|\right)\right\} d S . \\
+\frac{1}{2 \pi s_{1} s_{2} s_{3}} \int_{|\boldsymbol{\xi}|=1}\left\{\frac{\xi_{m} \xi_{l} \xi_{j}}{a \rho\left(a^{2}-p_{\xi}^{2}\right)^{2}} K_{j}(\boldsymbol{\xi}) H(a t-|\boldsymbol{x} \cdot \boldsymbol{\xi}|)\right. \\
\left.+\frac{\xi_{m}\left(\delta_{l j}-\xi_{l} \xi_{j}\right)}{b \rho\left(b^{2}-p_{\xi}^{2}\right)^{2}} K_{j}(\boldsymbol{\xi}) H(b t-|\boldsymbol{x} \cdot \boldsymbol{\xi}|)\right\} d S .
\end{gathered}
$$

From the above expression, we will prove the Eshelby property of constant strain for the interior domain. 


\subsection{The satisfaction of the radiation condition}

We will prove that the solutions (3.52) and (3.58) for the displacement and displacement gradient satisfy the radiation conditions, e.g.,

$\boldsymbol{u}(\boldsymbol{x}, t) \rightarrow 0, \quad$ as $\quad|\boldsymbol{x}| \rightarrow \infty$,

and

$\frac{\partial u_{l}(\boldsymbol{x}, t)}{\partial x_{m}} \rightarrow 0, \quad$ as $\quad|\boldsymbol{x}| \rightarrow \infty$

First we show that the expression for the displacement (3.52) automatically satisfies the radiation condition (3.59) which is equivalent to the boundary condition at infinity. We note that for $\boldsymbol{\zeta}=\boldsymbol{x} /|\boldsymbol{x}|$,

$$
\begin{gathered}
(\boldsymbol{x} \cdot \boldsymbol{\xi}) H\left(p_{\xi} t-|\boldsymbol{x} \cdot \boldsymbol{\xi}|\right)=|\boldsymbol{x}|(\boldsymbol{\zeta} \cdot \boldsymbol{\xi}) H\left(\frac{p_{\xi} t}{|\boldsymbol{x}|}-|(\boldsymbol{\zeta} \cdot \boldsymbol{\xi})|\right) \rightarrow p_{\xi} t(\boldsymbol{\zeta} \cdot \boldsymbol{\xi}) \delta(|(\boldsymbol{\zeta} \cdot \boldsymbol{\xi})|)=0 \\
\text { as } \quad|\boldsymbol{x}| \rightarrow \infty
\end{gathered}
$$

and

$$
\begin{aligned}
& \left(p_{\xi} t+\boldsymbol{x} \cdot \boldsymbol{\xi}\right) \operatorname{sgn}\left(p_{\xi} t+\boldsymbol{x} \cdot \boldsymbol{\xi}\right)-\left(p_{\xi} t-\boldsymbol{x} \cdot \boldsymbol{\xi}\right) \operatorname{sgn}\left(p_{\xi} t-\boldsymbol{x} \cdot \boldsymbol{\xi}\right) \\
& \quad=p_{\xi} t\left[\operatorname{sgn}\left(p_{\xi} t+\boldsymbol{x} \cdot \boldsymbol{\xi}\right)-\operatorname{sgn}\left(p_{\xi} t-\boldsymbol{x} \cdot \boldsymbol{\xi}\right)\right]+(\boldsymbol{x} \cdot \boldsymbol{\xi}) H\left(p_{\xi} t-|\boldsymbol{x} \cdot \boldsymbol{\xi}|\right) \\
& \quad \rightarrow 2 p_{\xi} t \operatorname{sgn}(\boldsymbol{\zeta} \cdot \boldsymbol{\xi}), \text { as } \quad|\boldsymbol{x}| \rightarrow \infty
\end{aligned}
$$

also

$$
\begin{gathered}
(a t+\boldsymbol{x} \cdot \boldsymbol{\xi}) \operatorname{sgn}(a t+\boldsymbol{x} \cdot \boldsymbol{\xi})-(a t-\boldsymbol{x} \cdot \boldsymbol{\xi}) \operatorname{sgn}(a t-\boldsymbol{x} \cdot \boldsymbol{\xi}) \\
\rightarrow 2 a t \operatorname{sgn}(\boldsymbol{\zeta} \cdot \boldsymbol{\xi}), \text { as }|\boldsymbol{x}| \rightarrow \infty, \\
(b t+\boldsymbol{x} \cdot \boldsymbol{\xi}) \operatorname{sgn}(b t+\boldsymbol{x} \cdot \boldsymbol{\xi})-(b t-\boldsymbol{x} \cdot \boldsymbol{\xi}) \operatorname{sgn}(b t-\boldsymbol{x} \cdot \boldsymbol{\xi}) \\
\rightarrow 2 b t \operatorname{sgn}(\boldsymbol{\zeta} \cdot \boldsymbol{\xi}), \text { as }|\boldsymbol{x}| \rightarrow \infty .
\end{gathered}
$$

By taking limit of $|\boldsymbol{x}| \rightarrow \infty$ in Eq. (3.52) and using (3.61) - (3.64), we proved 
the radiation condition (3.59) is satisfied, i.e.,

$\boldsymbol{u}(\boldsymbol{x}, t) \rightarrow 0 \quad$ as $\quad|\boldsymbol{x}| \rightarrow \infty$

Next, we will prove the solution of the displacement gradient (3.58) satisfies the radiation condition (3.60). In the expression of the displacement gradient (3.58), we note the following limits

$\delta\left(p_{\xi} t-|\boldsymbol{x} \cdot \boldsymbol{\xi}|\right)=\frac{1}{|\boldsymbol{x}|} \delta\left(\frac{p_{\xi} t}{|\boldsymbol{x}|}-|\boldsymbol{\zeta} \cdot \boldsymbol{\xi}|\right) \rightarrow 0, \quad$ as $\quad|\boldsymbol{x}| \rightarrow \infty$,

and

$H\left(p_{\xi} t-|\boldsymbol{x} \cdot \boldsymbol{\xi}|\right)=H\left(\frac{p_{\xi} t}{|\boldsymbol{x}|}-|\boldsymbol{\zeta} \cdot \boldsymbol{\xi}|\right) \rightarrow 0, \quad$ as $\quad|\boldsymbol{x}| \rightarrow \infty$

Similarly, we have

$H(a t-|\boldsymbol{x} \cdot \boldsymbol{\xi}|)=\rightarrow 0, \quad$ as $\quad|\boldsymbol{x}| \rightarrow \infty$,

and

$H(a t-|\boldsymbol{x} \cdot \boldsymbol{\xi}|)=\rightarrow 0, \quad$ as $\quad|\boldsymbol{x}| \rightarrow \infty$

Hence, by taking limit of $|\boldsymbol{x}|$ going to infinity in Eq. (3.58), and using the limits (3.66) - (3.69), we have the radiation condition (3.60),

$\frac{\partial u_{l}(\boldsymbol{x}, t)}{\partial x_{m}} \rightarrow 0, \quad$ as $\quad|\boldsymbol{x}| \rightarrow \infty$,

satisfied.

3.6 Solution for the particle velocity $\partial u_{l} / \partial t(\boldsymbol{x}, t)$ of a self-similarly subsonically expanding ellipsoidal inclusion

From the displacement solution (3.53), we obtain the expression for the particle velocity 


$$
\begin{aligned}
\frac{\partial u_{l}}{\partial t}(\boldsymbol{x}, t)= & \frac{-1}{4 \pi s_{1} s_{2} s_{3}} \int \frac{1}{|\boldsymbol{\xi}|=1}\left[\frac{\xi_{l} \xi_{j}}{\rho p_{\xi}^{2}}+\frac{\delta_{l j}-\xi_{l} \xi_{j}}{p_{\xi}^{2}-a^{2}}\right] K_{j}(\boldsymbol{\xi})(\boldsymbol{x} \cdot \boldsymbol{\xi}) \delta\left(p_{\xi} t-|\boldsymbol{x} \cdot \boldsymbol{\xi}|\right) \\
& \left.+\frac{2}{\rho}\left[\frac{\xi_{l} \xi_{j}}{\left(p_{\xi}^{2}-a^{2}\right)^{2}}+\frac{\delta_{l j}-\xi_{l} \xi_{j}}{\left(p_{\xi}^{2}-b^{2}\right)^{2}}\right] K_{j}(\boldsymbol{\xi}) \operatorname{sgn}\left(p_{\xi} t+\boldsymbol{x} \cdot \boldsymbol{\xi}\right)\right\} d S \\
+ & \frac{1}{2 \pi s_{1} s_{2} s_{3}} \int\left\{\frac{\xi_{l} \xi_{j}}{\rho\left(a^{2}-p_{\xi}^{2}\right)^{2}} K_{j}(\boldsymbol{\xi}) \operatorname{sgn}(a t+\boldsymbol{x} \cdot \boldsymbol{\xi})\right. \\
& \left.+\frac{\delta_{l j}-\xi_{l} \xi_{j}}{\rho\left(b^{2}-p_{\xi}^{2}\right)^{2}} K_{j}(\boldsymbol{\xi}) \operatorname{sgn}(b t+\boldsymbol{x} \cdot \boldsymbol{\xi})\right\} d S
\end{aligned}
$$

where Eqs. (3.24), (3.30), and (3.34) were used.

3.7 Solution for the particle acceleration $\partial^{2} u_{l} / \partial t^{2}(\boldsymbol{x}, t)$ of a self-similarly subsonically expanding ellipsoidal inclusion

From Eq. (3.58), we obtain the expression for the particle acceleration

$$
\begin{aligned}
\frac{\partial^{2} u_{l}}{\partial t^{2}}(\boldsymbol{x}, t)= & \frac{-1}{4 \pi s_{1} s_{2} s_{3}} \int_{|\boldsymbol{\xi}|=1}\left\{\frac{1}{\rho p_{\xi}}\left[\frac{\xi_{l} \xi_{j}}{p_{\xi}^{2}-a^{2}}+\frac{\delta_{l j}-\xi_{l} \xi_{j}}{p_{\xi}^{2}-b^{2}}\right] K_{j}(\boldsymbol{\xi})(\boldsymbol{x} \cdot \boldsymbol{\xi}) \delta^{\prime}\left(p_{\xi} t-|\boldsymbol{x} \cdot \boldsymbol{\xi}|\right)\right. \\
& \left.+\frac{4 p_{\xi}}{\rho}\left[\frac{\xi_{l} \xi_{j}}{\left(p_{\xi}^{2}-a^{2}\right)^{2}}+\frac{\delta_{l j}-\xi_{l} \xi_{j}}{\left(p_{\xi}^{2}-b^{2}\right)^{2}}\right] K_{j}(\boldsymbol{\xi}) \delta\left(p_{\xi} t-|\boldsymbol{x} \cdot \boldsymbol{\xi}|\right)\right\} d S . \\
+ & \frac{1}{\pi s_{1} s_{2} s_{3}} \int\left\{\frac{a \xi_{l} \xi_{j}}{\rho\left(a^{2}-p_{\xi}^{2}\right)^{2}} K_{j}(\boldsymbol{\xi}) \delta(a t-|\boldsymbol{x} \cdot \boldsymbol{\xi}|)\right. \\
& \left.+\frac{b\left(\delta_{l j}-\xi_{l} \xi_{j}\right)}{\rho\left(b^{2}-p_{\xi}^{2}\right)^{2}} K_{j}(\boldsymbol{\xi}) \delta(b t-|\boldsymbol{x} \cdot \boldsymbol{\xi}|)\right\} d S .
\end{aligned}
$$

3.8 The Eshelby property for the interior domain of the self-similarly subsonically expanding ellipsoidal inclusion

For any field point $\boldsymbol{x}$ inside the expanding inclusion, $s_{k}^{2} x_{k}^{2}<t^{2}$, by the Cauchy-Schwarz inequality, we have

$$
|\boldsymbol{x} \cdot \boldsymbol{\xi}|^{2}=\left|x_{k}^{2} \xi_{k}^{2}\right|^{2} \leq\left|\xi_{j}^{2} / s_{j}^{2}\right|\left|s_{k}^{2} x_{k}^{2}\right|<p_{\xi}^{2} t^{2}<\min \left\{a^{2} t^{2}, b^{2} t^{2}\right\}
$$


because, for subsonic motion of the expanding inclusion, we have

$1 / s_{i}<\min \{a, b\}$, for $i=1,2,3$,

so that

$p_{\xi}^{2}=\frac{\xi_{i}^{2}}{s_{i}^{2}}<\min \left\{a^{2}, b^{2}\right\}$

Then, for $\boldsymbol{x}$ inside the expanding inclusion, in Eq. (3.58), we have

$\delta\left(p_{\xi} t-|\boldsymbol{x} \cdot \boldsymbol{\xi}|\right)=0$,

$H\left(p_{\xi} t-|\boldsymbol{x} \cdot \boldsymbol{\xi}|\right)=H(a t-|\boldsymbol{x} \cdot \boldsymbol{\xi}|)=H(b t-|\boldsymbol{x} \cdot \boldsymbol{\xi}|)=1$.

Thus, Eq. (3.58) is reduced to

$$
\begin{aligned}
\frac{\partial u_{l}}{\partial x_{m}}(\boldsymbol{x}, t)= & \frac{-1}{4 \pi s_{1} s_{2} s_{3}} \int_{|\boldsymbol{\xi}|=1}\left\{\frac{2 \xi_{m}}{\rho p_{\xi}}\left[\frac{\xi_{l} \xi_{j}}{\left(p_{\xi}^{2}-a^{2}\right)^{2}}+\frac{\delta_{l j}-\xi_{l} \xi_{j}}{\left(p_{\xi}^{2}-b^{2}\right)^{2}}\right] K_{j}(\boldsymbol{\xi})\right. \\
& \left.+\frac{\xi_{m}}{\rho p_{\xi}^{3}}\left[\frac{\xi_{l} \xi_{j}}{p_{\xi}^{2}-a^{2}}+\frac{\delta_{l j}-\xi_{l} \xi_{j}}{p_{\xi}^{2}-b^{2}}\right] K_{j}(\boldsymbol{\xi})\right\} d S \\
+ & \frac{1}{2 \pi s_{1} s_{2} s_{3}} \int_{|\boldsymbol{\xi}|=1}\left[\frac{\xi_{m} \xi_{l} \xi_{j}}{a \rho\left(a^{2}-p_{\xi}^{2}\right)^{2}}+\frac{\xi_{m}\left(\delta_{l j}-\xi_{l} \xi_{j}\right)}{b \rho\left(b^{2}-p_{\xi}^{2}\right)^{2}}\right] K_{j}(\boldsymbol{\xi}) d S \\
= & \frac{1}{4 \pi s_{1} s_{2} s_{3}} \int_{|\boldsymbol{\xi}|=1} \frac{\xi_{m}}{\rho p_{\xi}^{3}}\left[\frac{\xi_{l} \xi_{j}\left(a+2 p_{\xi}\right)}{a\left(a+p_{\xi}\right)^{2}}+\frac{\left(\delta_{l j}-\xi_{l} \xi_{j}\right)\left(b+2 p_{\xi}\right)}{b\left(b+p_{\xi}\right)^{2}}\right] K_{j}(\boldsymbol{\xi}) d S
\end{aligned}
$$

The right hand side of the last equation is independent of the field point $\boldsymbol{x}$ and time $t$. Therefore, inside the subsonically expanding ellipsoidal inclusion, the displacement gradient, strain, and stress are all constants, and the wellknown property for static ellipsoidal inclusions (Eshelby, 1957) is extended to dynamics as pointed out by Buridge and Willis (1969).

Since (3.76) and (3.77) hold true inside the expanding ellipsoidal inclusion, for $\boldsymbol{x}$ in the interior domain of the expanding ellipsoidal inclusion, in Eq. 
(3.71), the integrals cancel each other, i.e.,

$$
\begin{aligned}
\frac{\partial u_{l}}{\partial t}(\boldsymbol{x}, t) & =\frac{-1}{2 \pi s_{1} s_{2} s_{3}} \int_{|\boldsymbol{\xi}|=1}\left\{\frac{1}{\rho}\left[\frac{\xi_{l} \xi_{j}}{\left(p_{\xi}^{2}-a^{2}\right)^{2}}+\frac{\delta_{l j}-\xi_{l} \xi_{j}}{\left(p_{\xi}^{2}-b^{2}\right)^{2}}\right] K_{j}(\boldsymbol{\xi})\right\} d S . \\
& +\frac{1}{2 \pi s_{1} s_{2} s_{3}} \int_{|\boldsymbol{\xi}|=1}\left[\frac{\xi_{l} \xi_{j}}{\rho\left(a^{2}-p_{\xi}^{2}\right)^{2}}+\frac{\delta_{l j}-\xi_{l} \xi_{j}}{\rho\left(b^{2}-p_{\xi}^{2}\right)^{2}}\right] K_{j}(\boldsymbol{\xi}) d S=0 .
\end{aligned}
$$

Hence, we have shown by the explicit calculation of the solution that inside the self-similarly subsonically expanding ellipsoidal inclusion the particle velocity is zero (lacuna), a result that can be also obtained by dimensional analysis alone, a question posted by J.R. Rice and answered by Markenscoff (2015) due to the fact that for a self-similarly expanding subsonic motion the hyperbolic system of the partial differential equations of motion becomes elliptic (as proved in Ni and Markenscoff, 2015) and analytic arguments. In general non self-similar motion of a spherical inclusion with nonzero initial radius, the focusing of the waves gives infinite stress at $r=0$ as discussed in Wills (1965) for time dependent eigenstrain, and in the solution in Markenscoff and Ni (2010), Eqs. (2.22) and (2.23) for an expanding inclusion analyzed in Part II of this work.

\section{Conclusion}

In this first part of the treatment of the self-similarly (subsonically) dynamically expanding Eshelby ellipsoidal inclusion we have obtained the field solution by the use of the Radon transform and the satisfaction of the initial conditions and the radiation condition at infinity. It constitutes the selfsimilar evolution of the inclusion singularity (jump discontinuity at the inclusion boundary) starting from zero dimension. The field solutions for the displacement gradient and particle velocity were presented. It was shown explicitly that the solution for the displacement gradient in the interior domain of expanding ellipsoid is constant, thus extending the Eshelby property to the 
self-similarly expanding ellipsoid, and obtaining the static Eshelby tensor as a limiting case of the self-similar dynamic one for the sphere (in Part II of this treatment).

The general expression for the field solution of the displacement gradient obtained in this first part of the analysis constitutes the basis for obtaining the Dynamic Eshelby Tensor for the expanding spherical inclusion in the part II.

\section{Acknowledgement}

This work was supported by National Science Foundation grant CMS 1129888.

\section{References}

Backus, G. and Mulcahy, M. (1976) Moment tensors and other phenomenological descriptions of seimic sources-I. Continuous displacement. Geophys. J. R. astr. Soc., 46, 341-361.

Barenblatt, G.I. (1979) Scaling, Self-Similarity, and Intermediate Asymptotics(1st Russian edition Gidrometeoizdat, Leningrad, 1978; 2nd Russian edition, Gidrometeoizdat, Leningrad, 1982). Plenum, New York, London.

Barenblatt, G.I. (1996) Scaling, Self-Similarity, and Intermediate Asymptotics, Cambridge University Press.

Bluman, G.W. and Kumei, S. (1989) Symmetries and Differential Equations, Spring-Verlag.

Burnley, P.C. and Green II. H.W. (1989) Stress dependence of the mechanism of the olivine-spinel transformation. Nature, 338, 753-756.

Burridge, R. and Willis, J.R. (1969) The self-similar problem of the expanding elliptical crack in an anisotropic solid. Proc. Camb. Phil. Soc., 66, 443468.

Dundurs, J. (1967). On the interaction of a screw dislocation with inhomo- 
geneities. Recent advances in engineering science, Vol. 2, 222C233. Eringen, A.C., Gordon and Breach.

Eshelby, J.D. (1957) The determination of the elastic field of an ellipsoidal inclusion and related problems. Proc. Roy. Soc. London, A241, 376-396.

Eshelby, J.D. (1959) Scope and limitations of the continuum approach. In Internal Stresses and Fatigue in Metals, eds. G.N. Rassweiler and W.I. Grube, 41-58, Elservier, Amsterdam.

Eshelby, J.D. (1961) Elastic inclusion and inhomogeneities. Prog. Solid Mech., 2, 89-140.

Ferguson, C.C. (1979) Intersections of ellipsoids and planes of arbitrary orientation and position. Mathematical Geology, 11, No. 3, 329-336.

Gel'fand, I.M. and Shilov, G.E. (1964) Generalized Functions, Vol. I, Academic Press.

Green II, H.W. and Burnley, P.C. (1989). A new self-organizing mechanism for deep-focus earthquakes. Nature, 341(6244), 733-737.

John, F. (1955) Plane Waves and Spherical Means, Interscience Publishers, Ins., New York.

Li, S. and Wang, G. (2008) Introduction to Micromechanics and Nanomechanics, World Scientific.

Ludwig, D. (1966) The Radon transform on Euclidean space. Communs. pure appl. Math., 19, 49-81.

Markenscoff, X. (2015) The properties of the self-similarly expanding Eshelby inclusions and application to the dynamic "Hill jump conditions". J. Math. Mech. solids, (online 1081286515607094).

Markenscoff, X. and Ni, L. (2010) The energy-release rate and self-force of dynamically expanding spherical and plane inclusion boundaries with dilatational eigenstrain. J. Mech. Phys. Solids, 58, 1C11.

Markenscoff, X. and Ni, L. (2012) Driving forces on expanding strips with general transformation strain. Int. J. Engineering Science, 61, 94-102.

Michelitsch, T.M., Gao, H., and Levin, V.M. (2003) Dynamic Eshelby tensor and potential for ellipsoidal inclusion. Proc. R. Soc. Lond. A. 459, 863-890. Mikata, Y. and Nemat-Nasser, S. (1990) Elastic field due to a dynamically 
transforming spherical inclusion. J. Appl. Mech. 57(4), 845-849.

Ni, L. and Markenscoff, X. (2015) On self-similarly expanding Eshelby inclusions: spherical inclusions with dilatational eigenstrain. Mechanics of $\mathrm{Ma}^{-}$ terials, 90, 30-36.

Rice, J.R. (1980) The mechanics of earthquake rupture. In Physics of the Earth's Interior, Proc. Int. School of Physics "E. Fermi", Course 78, 1979, Eds. A.M. Dziewonski and E. Boschi, Italian Physical Society North Holland Publ. Co.

Wang, C.Y. and Achenbach, J.D. (1994) Elastodynamic fundamental solutions for anisotropic solids. Geophysical Journal International, 118, 384-392. Wang, Y.C. and Lakes, R.S. (2005) Composites with inclusions of negative bulk modulus: extreme damping and negative Poisson's ratio. Journal of Composits Materials, 39, No. 18, 1745-1758.

Wheeler, L.T. and Sternberg, E. (1968) Some Theorems in classical elastodynamics. Arch. Rational Mech Anal., 31, 51-90.

Willis, J.R. (1965) Dislocations and inclusions. J. Mech. Phys. Solids, 13, 337-395.

Willis, J.R. (1973) Self-similar problems in elastodynamics. Phil. Trans. Roy. Soc. Lond., A, 274, 435-491.

Yang, S.Y., Escobar, J. and Clifton, R.J. (2009) Computational modeling of stress-wave-induced martensitic phase transformations in NiTi. Mathematics and Mechanics of Solids, 14, 220-257. 